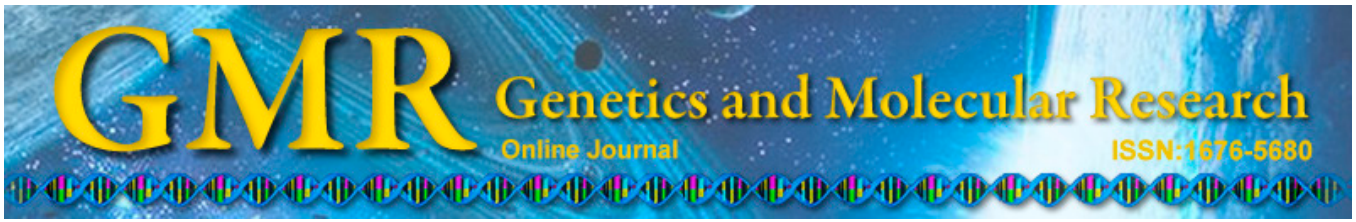

\title{
Synergistic effect of BMP9 and TGF- $\beta$ in the proliferation and differentiation of osteoblasts
}

\author{
X.L. Li ${ }^{1}$, Y.B. Liu ${ }^{2}$, E.G. Ma ${ }^{3}$ W.X. Shen ${ }^{4}$, H. Li ${ }^{5}$ and Y.N. Zhang ${ }^{1}$ \\ ${ }^{1}$ The Cadre Ward, The Second Affiliated Hospital, Harbin Medical University, \\ Harbin, China \\ ${ }^{2}$ Department of Cardiology, The Second Affiliated Hospital, \\ Harbin Medical University, Harbin, China \\ ${ }^{3}$ Department of Urinary Surgery, Harbin First Hospital, Harbin, China \\ ${ }^{4}$ Medical Oncology of Cardiology, The Second Affiliated Hospital, \\ Harbin Medical University, Harbin, China \\ ${ }^{5}$ The Professional Judicial Police College of Heilongjiang, Health Clinic, \\ Harbin, China \\ Corresponding author: Y.N. Zhang \\ E-mail: lixinglu3@126.com
}

Genet. Mol. Res. 14 (3): 7605-7615 (2015)

Received July 17, 2014

Accepted January 29, 2015

Published July 13, 2015

DOI http://dx.doi.org/10.4238/2015.July.13.4

\begin{abstract}
We investigated the synergistic effect of bone morphogenetic protein 9 (BMP9) and transforming growth factor (TGF)- $\beta$ in the transformation of mesenchymal stem cells into osteoblasts. We evaluated the effect of BMP9 and TGF- $\beta$ on the induction of osteoblast formation. Mitogen-activated protein kinase (MAPK) pathway-related proteins such as p38, extracellular receptor kinase $1 / 2$, and c-Jun N-terminal kinase (JNK) were analyzed. The interactions between TGF-Smad and BMP-MAPK were also studied. BMP9 alone induced the differentiation of mesenchymal stem cells (MSCs) into osteoblasts and enhanced phosphorylation of $\mathrm{p} 38$, extracellular receptor kinase $1 / 2$, and JNK. TGF- $\beta$ alone failed to induce transformation, but could increase the effect of
\end{abstract}


BMP9. In this process the activation of Smad resulted in activation of the JNK pathway in the MAPK pathway. BMP9 induced osteogenesis of MSC differentiation through the MAKP pathway, while TGF- $\beta$ contributed to BMP9 enhancement through the Smad-JNK pathway.

Key words: Bone morphogenetic protein 9; Cell differentiation; Mesenchymal stem cells; Mitogen activated protein kinase; Signal transduction pathway

\section{INTRODUCTION}

Mesenchymal stem cells (MSCs) are mesoderm pluripotent stem cells that exist in the marrow cavity and show multi-directional differentiation potential. They can differentiate into muscle cells, bone cells, or cartilage cells (Luther et al., 2011). MSCs are often used as seed cells in the bone cell regeneration therapy for fracture trauma repair (Li et al., 2012). Multiple types of relevant stimulating factors are needed to induce MSC differentiation, of which bone morphogenetic proteins (BMPs) play the most important role. BMPs belong to the transforming growth factor (TGF) superfamily, which contains more than 20 subtypes. Among these, BMP 2, 4, 6, and 7 were found to be related to osteoblast generation (Williams et al., 2011; Zhao et al., 2013; Wang et al., 2013). In recent years, BMP9, as a new class of BMP, has been widely studied for its strong impact on the induction of MSC differentiation. However, its mechanism and correlation with traditional transforming factors remain unknown (Xu et al., 2012). In this study, we found that BMP9 mainly affects MSC differentiation through the mitogen-activated protein kinase (MAPK) pathway. Furthermore, BMP9 was shown to interact with the traditional classic stimulating factor TGF- $\beta$-Smad pathway.

\section{MATERIAL AND METHODS}

\section{Materials}

The mouse MSC cell line C3H10T1/2 was purchased from American Type Culture Collection (Manassas, VA, USA). Adenovirus vector AdBMP9 and the related empty vector AdGF were constructed by GeneCopoela (Harbin, China). Monoclonal antibodies for phosphorylated p38, extracellular receptor kinase 1/2 (ERK1/2), and c-Jun N-terminal kinase (JNK) were purchased from R\&D Systems (Minneapolis, MN, USA). Monoclonal Smad 2/3 antibody was purchased from Cell Signaling Technology (Danvers, MA, USA).

\section{Methods}

\section{Cell culture and adenovirus transfection}

C3H10T1/2 cells were maintained in high glucose-Dulbecco's modified Eagle medium supplemented with $10 \%$ fetal bovine serum in a humid atmosphere containing $5 \% \mathrm{CO}_{2}$ at $37^{\circ} \mathrm{C}$. Cells in exponential growth phase were used for experiments. Ad-BMP9 or empty vector was loaded when the cell density reached $30-40 \%$. 


\section{Cell stimulation and intervention}

\section{Kinase intervention}

Different kinase inhibitors (SP600125, JNK inhibitor; SB203580, p38 kinase inhibitor; PD98059, ERK1/2 inhibitor) were used to pretreat the cells for $4 \mathrm{~h}$. Next, Ad-BMP9 was added for the following tests.

\section{Alkaline phosphatase (ALP) activity detection}

ALP activity was evaluated using an ALP detection kit (Shanghai Bohu, China). Briefly, the differentiated cells were washed with sterile phosphate-buffered saline and lysed. The supernatant was used for detection.

\section{OPN and COLI detection}

mRNA was extracted and reverse transcription (RT)-polymerase chain reaction (PCR) was used for detection.

\section{TGF- $\beta$ co-stimulation}

C3H10T1/2 cells in the exponential growth phase were grouped as follows: group A, BMP9 stimulation, Ad-BMP9 was transfected into the cells based on a $25 \%$ infection rate; group B, TGF- $\beta$ stimulation, $10 \mathrm{ng} / \mathrm{mL}$ recombinant TGF- $\beta$ (Peprotech Company, Rocky Hill, NJ, USA) was added to the cells; group C, BMP9+TGF- $\beta$ co-stimulation, the two reagents were added to the cells simultaneously; and group D, control, empty vector was transfected into the cells at the same dose as applied for group A.

Cells in group C were further treated with the Smad 2/3 inhibitor SB525334 and the phosphorylation level of BMP-MAPKs-related kinase was evaluated.

\section{Detection and analysis}

\section{Western blot}

Expression of p-JNK, p-ERK1/2, p-p38, and Smad 2/3 was analyzed by western blotting. Cells were washed with ice-cold phosphate-buffered saline and then lysed in RIPA buffer (25 mM Tris- $\mathrm{HCl}, \mathrm{pH} 7.5,2.5 \mathrm{mM}$ ethylenediaminetetraacetic acid, $10 \mathrm{mM}$ sodium pyrophosphate, $50 \mathrm{mM} \mathrm{NaF}, 137 \mathrm{mM} \mathrm{NaCl}, 100 \mu \mathrm{M} \mathrm{Na}_{3} \mathrm{VO}_{4}, 1 \%$ Triton X-100, $10 \%$ glycerol, $1 \%$ deoxycholic acid, $0.1 \%$ sodium dodecyl sulfate, and fresh $0.1 \mathrm{mM}$ phenylmethylsulfonyl fluoride, and $10 \mathrm{mg} / \mathrm{mL}$ leupeptin). Cell lysates were prepared by scraping, sonication, and centrifugation. Cellular protein concentrations were determined using the BCA protein assay. Cell lysates were subjected to sodium dodecyl sulfate-polyacrylamide gel electrophoresis under reducing conditions, and the protein bands then transferred to a nitrocellulose membrane. The membrane was blocked for $2 \mathrm{~h}$ at room temperature with $5 \%$ skim milk, and then incubated for $1 \mathrm{~h}$ at room temperature with primary antibodies, followed by incubation for $1 \mathrm{~h}$ with the secondary antibody, conjugated horseradish peroxidase. The membrane was then devel- 
oped and exposed in the dark room. After that an image of all blotting bands were captured by UV-transilluminator and relative density of all bands was analyzed by Quantity One software.

PCR

Real-time RT-PCR was used to verify differential expression of the $O P N$ and $C O L I$ genes. cDNA was synthesized using reverse transcriptase (TaKaRa, Shiga, Japan), oligo (dT) primers, and $1 \mu \mathrm{g}$ RNA from the same samples as those used in the microarray. The primers used are listed in Table 1. Each real-time RT-PCR (in $20 \mu \mathrm{L}$ ) contained 2.5X SYBR Green Realtime PCR Master Mix (TIANGEN, Beijing, China), $0.5 \mu \mathrm{M}$ of each primer, and $0.5 \mu \mathrm{L}$ template cDNA. The cycling conditions consisted of an initial, single cycle of $2 \mathrm{~min}$ at $94^{\circ} \mathrm{C}$, followed by 40 cycles of $15 \mathrm{~s}$ at $94^{\circ} \mathrm{C}, 20 \mathrm{~s}$ at $63^{\circ} \mathrm{C}$, and $30 \mathrm{~s}$ at $68^{\circ} \mathrm{C}$. PCR amplifications were performed in 3 duplicates for each sample. The primer used are shown in Table.

\begin{tabular}{ll}
\multicolumn{1}{c}{ Table 1. PCR primers. } \\
\hline Gene & Sequence \\
\hline$O P N$ & F: 5'-3': CTTTCACTCCAATCGTCCCTAC \\
$O P N$ & R: 5'-3': CTGCCCTTTCCGTTGTTGTC \\
$C O L I$ & F: 5'-3': AGCGAAGAACTCATACAGCCG \\
$C O L I$ & R: 5'-3': TGCCCGTCTCCTCATCCA \\
\hline
\end{tabular}

\section{Flow cytometry}

Cells were stained by propidium iodide and flow cytometry was used to detect fluorescence intensity. MODFIT 2.0 was used for cell proliferation analysis (Verity Software House, Topsham, ME, USA).

\section{Statistical analysis}

Numerical data are reported as means and standard errors (SE). Differences between means were analyzed using the Student $t$-test or one-way analysis of variance. All statistical analyses were performed using the SPSS 17.0 software (Chicago, IL, USA). P values $<0.05$ were considered to be statistically significant.

\section{RESULTS}

\section{BMP9 promotes MAPKs signaling pathway-related kinase phosphorylation}

Western blotting was used to detect MAPK signaling pathway changes after Ad-BMP9 transfection (Figure 1). Compared with the empty transfection group, the phosphorylation level of JNK, p38, and ERK1/2 was significantly increased $(\mathrm{P}<0.05)$ after Ad-BMP9 transfection.

\section{Effects of MAPK-related kinase inhibition}

\section{Early-stage osteogenesis ALP activity analysis}

Relevant inhibitors of JNK, p38, and ERK1/2 were used to treat BMP9-stimulated 
MSCs, and ALP activity was detected after 6 days to evaluate osteogenic activity. Specially, ALP failed to change after JNK or p38 inhibition, while it increased significantly after ERK1/2 inhibition $(\mathrm{P}<0.05)$ (Table 2).

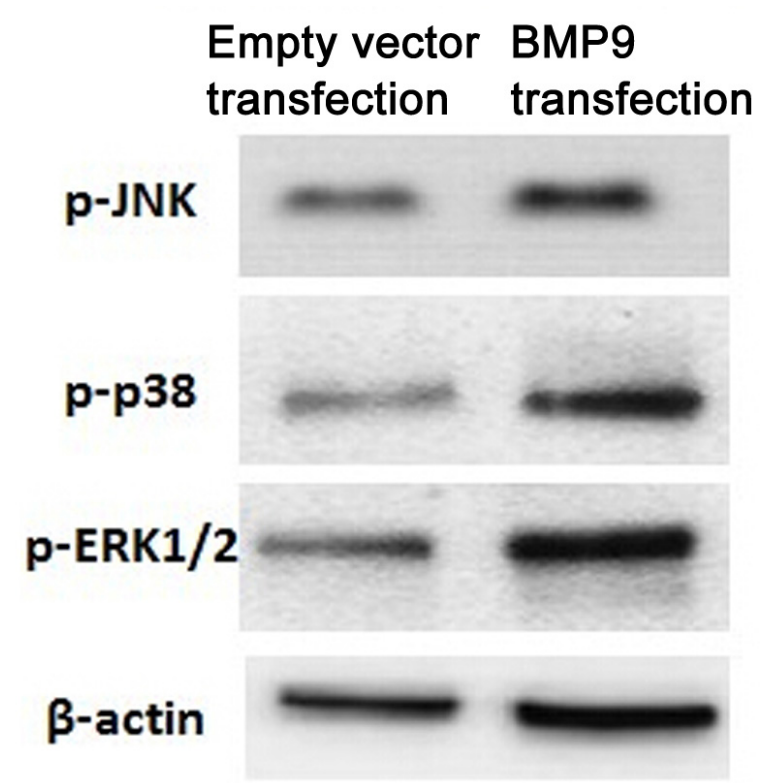

Figure 1. BMP9 promoted MAPK signaling pathway-related kinase phosphorylation. p-JNK, p-p38, and p-ERK1/2 level increased in BMP9-transfected cells.

Table 2. Early-stage osteogenesis ALP activity analysis $\left(\mathrm{x} 10^{3}\right)$.

\begin{tabular}{|c|c|c|c|c|}
\hline Time & Untreated & JNK inhibition & p38 inhibition & ERK1/2 inhibition \\
\hline D3 & $25.25 \pm 0.62$ & $25.47 \pm 0.66$ & $25.19 \pm 0.59$ & $39.22 \pm 1.29^{*}$ \\
\hline D6 & $132.48 \pm 9.16$ & $130.92 \pm 9.03$ & $131.89 \pm 9.21$ & $264.31 \pm 12.34$ \\
\hline
\end{tabular}

\section{Late-stage osteogenesis expression analysis}

Relevant inhibitors of JNK, p38, and ERK1/2 were used to treat cells for 5 days, and $O P N$ and $C O L I$ expression level was evaluated by real-time PCR. Compared with the untreated group, $O P N$ and $C O L I$ expression decreased under JNK inhibition, but increased after p38 or ERK1/2 inhibitor treatment $(\mathrm{P}<0.05)$ (Figure 2).

\section{TGF- $\beta$ co-stimulation effect analysis}

\section{Early-stage osteogenesis ALP expression analysis}

After 6 days of treatment of BMP9, TGF- $\beta$, or combined stimulation, ALP activity in cells was detected. BMP9 alone or in combination with TGF- $\beta$ increased ALP activity, while stimulation with TGF- $\beta$ alone did not show a similar effect $(\mathrm{P}<0.05)$ (Table 3$)$. 
A

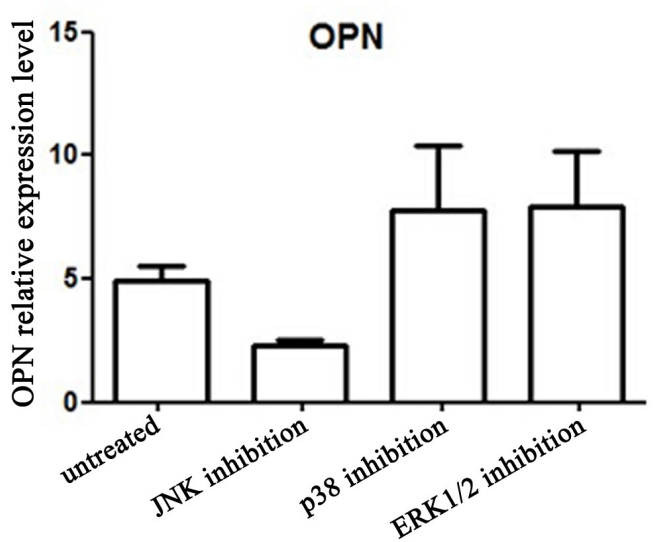

B

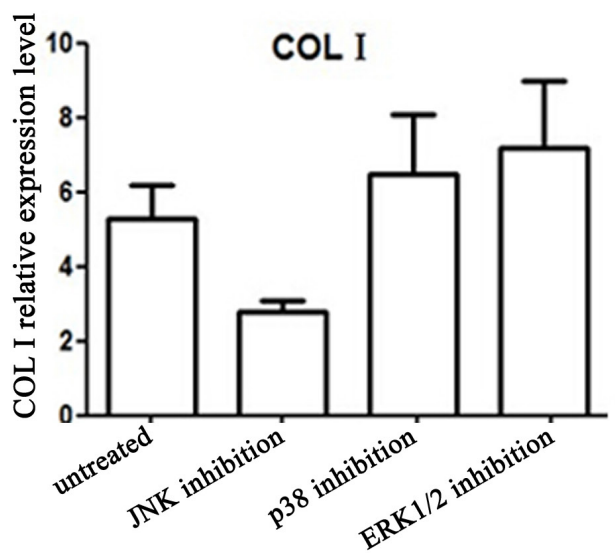

Figure 2. $O P N$ and $C O L I$ expression after MAPK inhibition. All groups were stimulated by BMP9. A. $O P N$ expression after MAPK inhibition. All groups were stimulated by BMP9. OPN expression was decreased after JNK was inhibited, but increased after p38 or ERK1/2 treatment. B. COLI expression after MAPK inhibition. All groups were stimulated by BMP9. COLI expression decreased after JNK inhibition, but increased after p38 or ERK1/2 treatment.

Table 3. ALP activity detection after BMP9, TGF- $\beta$, or combined stimulation $\left(\mathrm{x} 10^{3}\right)$.

\begin{tabular}{|c|c|c|c|c|}
\hline Time & Control & BMP9 stimulation & TGF- $\beta$ stimulation & Co-stimulation \\
\hline D3 & $3.21 \pm 0.42$ & $25.25 \pm 0.62 *$ & $2.98 \pm 0.38$ & $24.29 \pm 0.48^{*}$ \\
\hline D6 & $22.68 \pm 1.06$ & $132.48 \pm 9.16^{*}$ & $22.14 \pm 1.12$ & $128.46 \pm 11.22 *$ \\
\hline
\end{tabular}

\section{Late-stage osteogenesis expression analysis}

After 12 days of treatment with BMP9, TGF- $\beta$, or a combination of these factors, $O P N$ and $C O L I$ expression levels were detected. TGF- $\beta$ alone or combined with BMP9 increased ALP activity. Stimulation with BMP9 alone also increased gene expression, but to a lower extent than did TGF- $\beta(\mathrm{P}<0.05)$ (Figure 3$)$.

\section{Cell proliferation analysis}

After $72 \mathrm{~h}$ of BMP9, TGF- $\beta$, or combination treatment, proliferation of MSCs was detected by flow cytometry. Compared with the BMP9 alone treatment, a higher proportion of cells in the G0/G1 stage appeared after co-stimulation. This suggests that cell proliferation activity was higher after TGF- $\beta$ and BMP9 combination treatment $(\mathrm{P}<0.05)$ (Figure 4).

\section{Signaling pathway and related kinase expression analysis after BMP9, TGF- $\beta$, or combination treatment}

After combination treatment with BMP9 and TGF- $\beta$, the Smad 2/3 pathway was acti- 
vated. This suggests that TGF- $\beta$ promoted Smad $2 / 3$ pathway expression through co-stimulation. Furthermore, combination treatment resulted in higher p-JNK expression and inhibition of p-p38 expression. After further treatment with the Smad 2/3 inhibitor SB525334, Smad 2/3 expression was downregulated and the above-mentioned changes also weakened or disappeared. This indicated that co-stimulation can activate the TGF- $\beta$-Smad $2 / 3$ pathway, inhibit p38 activation, and promote JNK activity in the MAPK pathway (Figure 5).

A

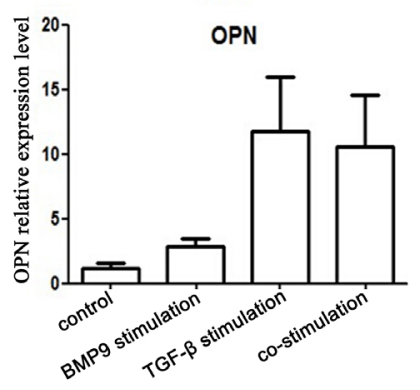

B

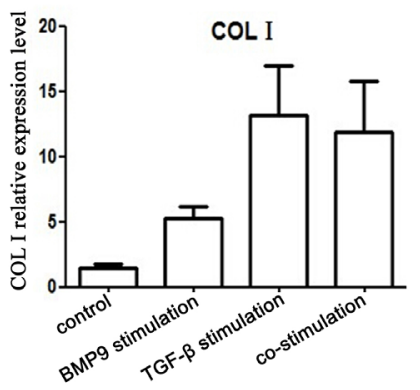

Figure 3. $O P N$ and $C O L I$ expression after BMP9, TGF- $\beta$, or combined stimulation. Control group was treated with empty vector-transfected cells; BMP9 treatment group was transfected with BMP9 adenovirus; TGF- $\beta$ treatment group was treated with $5 \mathrm{ng} / \mathrm{mL}$ TGF- $\beta$; BMP9+ TGF- $\beta$ treatment group involved combined stimulation. A. $O P N$ expression after BMP9, TGF- $\beta$ or combined stimulation. Control group was treated with empty vector-transfected cells; BMP9 treatment group was transfected with BMP9 adenovirus; TGF- $\beta$ treatment group was treated with $5 \mathrm{ng} / \mathrm{mL}$ TGF- $\beta$; BMP9+ TGF- $\beta$ treatment group involved combined treatment. $O P N$ expression elevated in the entire 3 stimulation groups and was much higher in the TGF- $\beta$ treated groups. B. COLI expression after BMP9, TGF- $\beta$ or combined stimulation. Control group was treated with empty vector-transfected cells; BMP9 treatment group was transfected with BMP9 adenovirus; TGF- $\beta$ treatment group was treated with $5 \mathrm{ng} / \mathrm{mL}$ TGF- $\beta$; BMP9+ TGF- $\beta$ treatment group involved combined stimulation. $C O L I$ expression elevated in all 3 stimulation groups and was much higher in the TGF- $\beta$-treated groups.

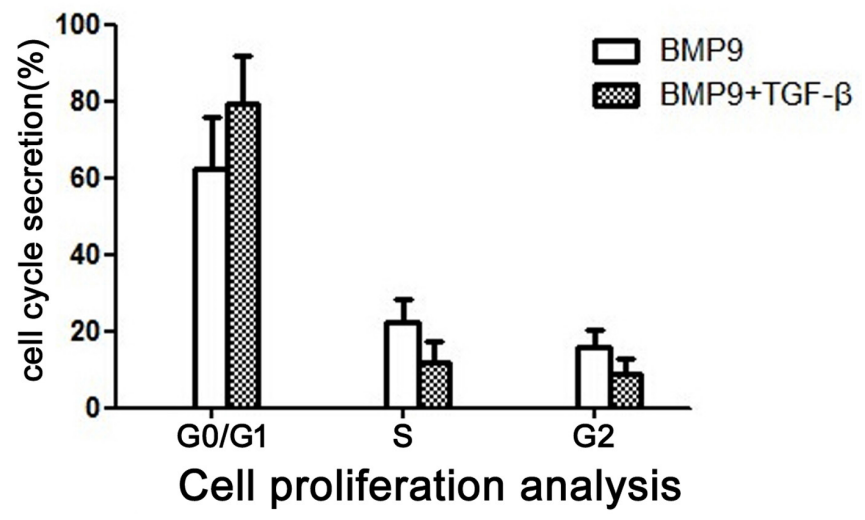

Figure 4. Cell proliferation activity analysis after BMP9 and TGF- $\beta$ co-stimulation. Cell cycle distribution directly represents cell proliferation activity. The higher proportion of G0/G1-stage cells indicated cells in mitosis, while the remaining cells were in S and G2. Cells treated with TGF- $\beta$ co-stimulation exhibited a higher proportion of cells in the G0/ G1 stage than BMP9 single treatment, revealing that TGF- $\beta$ had good auxiliary function in promoting cell proliferation. 


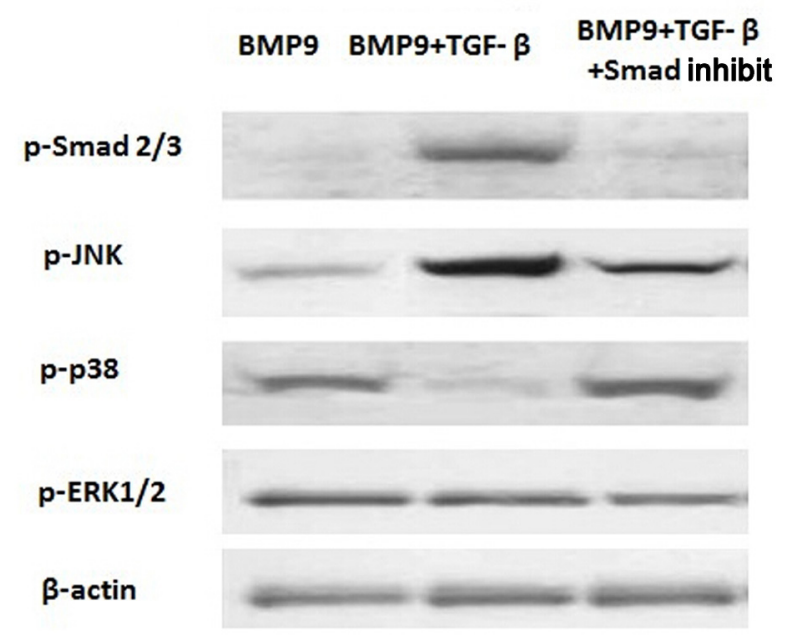

Figure 5. Signaling pathway and related kinase expression analysis after BMP9, TGF- $\beta$ or combined stimulation. Smad 2/3 activation enhanced JNK phosphorylation and weakened p-38 phosphorylation. BMP9, cells treated by BMP9 adenovirus; BMP9+TGF- $\beta$, cells treated by BMP9 and TGF- $\beta$; BMP9+TGF- $\beta+$ Smad inhibitor, cells treated with BMP9 adenovirus, TGF- $\beta$ and Smad inhibitor.

\section{DISCUSSION}

The induction of MSCs to become osteoblasts is an important process in bone tissue engineering, and identifying the appropriate factors is critical. Previous studies suggested that BMP2, 4, 7, and 9 can induce the differentiation of MSCs into osteoblasts. Among these, BMP9 showed the most powerful inductive effect. It can not only induce heterotopic ossification but also induce MSCS differentiation during early stages, indicating its value in research and clinical applications. Thus, we investigated BMP9 in this study.

BMP is thought to affect MSCs mainly through Smad signaling pathways, as it is a member of the TGF superfamily. BMPs first combine with TGF- $\beta$ receptors, activate the corresponding Smad pathway such as Smad 1, 5, and 8, and phosphorylate these factors, triggering transcription factors in the nuclei to cause relevant biological effects (Augello and De Bari, 2010). In addition to the classic signaling pathways, some reports suggested that BMPs can also act through an independent-Smad pathway such as the MAPK pathway (Hu et al., 2013). In contrast, other studies suggested that though BMPs showed strong effects in promoting cell differentiation, animal experimental results were not ideal. Animal experiments showed that under BMP9 stimulation, callus formation time was longer and the intensity was poor (Wu et al., 2010). In our study, we found that BMP9 can stimulate the proliferation of MSCs to some extents and is effective in promoting the transformation of MSCs, whereas MSC proliferation after transforming finalization requires other auxiliary stimulating factors.

In addition to the BMPs, TGF- $\beta 1$, as the common factor in the TGF family, plays an important role in tissue trauma and repair (Lamplot et al., 2013). However, stimulation with TGF- $\beta 1$ alone cannot induce the differentiation of MSCs into osteoblasts, limiting its research and application value in the tissue engineering of MSC transformation and proliferation (Brady et al., 2014). Considering the powerful promoting effect of TGF- $\beta 1$ on mesenchymal 
cell proliferation, we stimulated the osteoblasts directly using TGF- $\beta 1$ and found that osteoblast proliferation was significantly improved and its effect on ossein synthesis was also increased. These results suggest the importance of TGF- $\beta 1$ in trauma repair after osteoblast finalization. TGF- $\beta 1$ and BMP9 show complementary effects in trauma repair (Patel et al., 2013).

To investigate the mechanism of the synergic effect of these 2 important stimulating factors and the interaction between signaling pathways, we further examined the cell signaling pathway. Though no significant change was found in the total expression of $\mathrm{p} 38$, ERK1/2, and JNK in the MAPK pathway, their phosphorylation levels increased significantly after MSCs were transfected with Ad-BMP9. Therefore, the p38, ERK1/2, and JNK pathways in MSCs were activated under BMP9 stimulation (Madl et al., 2014). To further explore the different effects among the 3 pathways, we used corresponding pathway protein or kinase blockers to inhibit their expression. We selected different indices in the early and late stage of osteogenesis to evaluate the stimulus or blocking effect. ALP was selected as an early index as it is present during the initial stages of osteogenesis and is the foundation of calcium salt deposit adhesion during the late stage. In addition, $C O L I$ and $O P N$ were used as late-stage indices, as the former is a marker of local connective tissue formation, while the latter is a key indicator of bone matrix mineralization (Herencia et al., 2012; Liu et al., 2012; Luchetti et al., 2014; Madl et al., 2014).

After treatment of cells with the JNK kinase blocker, ALP level did not change, while $O P N$ and $C O L I$ expression was significantly reduced. Because ALP corresponds to early osteogenesis differentiation and $C O L I$ and $O P N$ represent late-stage differentiation, the results suggest that the BMP9-JNK pathway can facilitate osteogenesis during the late stage. Osteogenesis was not weakened, but rather strengthened after treatment of cells with the inhibitor of ERK1/2 kinase or p38 kinase. $O P N$ and $C O L I$ expression increased significantly after p38 inhibition, indicating the inhibitory role of the BMP9-p38 pathway during late-stage osteogenesis. While blockage with ERK1/2 kinase resulted in overexpression of ALP, OPN and COLI indicated the multiple promoting roles of the BMP9-ERK1/2 pathway in the 2 stages of osteogenesis.

After preliminary assessment of the effect of BMP9 on signaling pathways, we investigated the effect of TGF- $\beta$ alone or combination stimulus. Our results confirmed that application of TGF- $\beta$ alone did not induce MSC differentiation, while combination treatment that included TGF- $\beta$ showed a better late osteogenesis index and cell proliferation ability compared to BMP9 alone. These results confirmed the synergic effect of the 2 factors. The Smad 2/3 pathway was also activated in the combined treatment group in further cell signaling pathway analysis, suggesting synergic promotion. The TGF- $\beta$-Smad signaling pathway is the most important intracellular signal transduction medium. Smad 2/3 can activate a large number of target genes such as collagen I, cell cycle regulator p15, and p21, among others (De Gorter et al., 2011), as well as cause cell gathering and osteogenesis (Bharadwaj et al., 2013; Gao et al., 2013; Knight and Hankenson, 2013). In this study, though single application of TGF- $\beta$ failed to result in MSC transformation, strengthened late-stage osteogenesis and cell proliferation abilities were observed under combined use of TGF- $\beta$. This compensated for the limited effect of BMP9 alone stimulation in late-stage osteogenesis. Thus, early BMP9 stimulation combined with late TGF- $\beta$ stimulation can best facilitate the osteogenic effect. The difference in treatment time depends on the characteristics of the 2 factors. Interestingly, we found that MAPK negatively regulated kinase p38 and positively regulated kinase JNK after Smad $2 / 3$ activation. This explained the mechanism of TGF- $\beta$ promotion of further osteoblast maturation and proliferation in the late stage.

In summary, several phases, including differentiation, proliferation, and osteogenesis, 
occur during MSC differentiation to osteoblasts and promote subsequent tissue repair. Our results confirmed the strong effect of BMP9 in inducing MSC osteogenesis. In addition to the classic BMP9-Smad 1/4/5/9 pathways, this inducing effect may be associated with MAPK pathway activation by BMP9. The BMP9-JNK pathway was the most import induction pathway and played an important role in MSC late-stage differentiation. BMP9 also activated p38 and ERK1/2, but these pathways appeared to have negative feedback in facilitating differentiation. The BMP9-p38 pathway inhibited late-stage osteogenesis differentiation and BMP9-ERK1/2 blocked both the early and late stages. Activation of TGF- $\beta$-Smad inhibited late phosphorylation of p38 and enhanced expression of JNK; blockage of the Smad 2/3 pathway weakened these effects. This indicated that early BMP9 stimulation combined with late TGF- $\beta$ stimulation can be used as a new medication mode for facilitating bone tissue repair. However, in vivo experiments are needed to further investigate our results in an animal bone wound-healing model.

\section{Conflicts of interest}

The authors declare no conflict of interest.

\section{REFERENCES}

Augello A and De Bari C (2010). The regulation of differentiation in mesenchymal stem cells. Hum. Gene Ther. 21: 1226-1238.

Bharadwaj S, Liu G, Shi Y, Wu R, et al. (2013). Multipotential differentiation of human urine-derived stem cells: potential for therapeutic applications in urology. Stem Cells. 31: 1840-1856.

Brady K, Dickinson SC, Guillot PV, Polak J, et al. (2014). Human fetal and adult bone marrow-derived mesenchymal stem cells use different signaling pathways for the initiation of chondrogenesis. Stem Cells Dev. 23: 541-554.

De Gorter DJ, van Dinther M, Korchynskyi O and ten Dijke P (2011). Biphasic effects of transforming growth factor beta on bone morphogenetic protein-induced osteoblast differentiation. J. Bone Miner. Res. 26: 1178-1187.

Gao Y, Huang E, Zhang H, Wang J, et al. (2013). Crosstalk between Wnt/beta-catenin and estrogen receptor signaling synergistically promotes osteogenic differentiation of mesenchymal progenitor cells. Plos One 8: e82436.

Herencia C, Martinez-Moreno JM, Herrera C, Corrales F, et al. (2012). Nuclear translocation of $\beta$-catenin during mesenchymal stem cells differentiation into hepatocytes is associated with a tumoral phenotype. Plos One 7: e34656.

Hu N, Jiang D, Huang E, Liu X, et al. (2013). BMP9-regulated angiogenic signaling plays an important role in the osteogenic differentiation of mesenchymal progenitor cells. J. Cell Sci. 126: 532-541.

Knight MN and Hankenson KD (2013). Mesenchymal stem cells in bone regeneration. Adv. Wound Care (New Rochelle) 2: 306-316.

Lamplot JD, Denduluri S, Liu X, Wang J, et al. (2013). Major signaling pathways regulating the proliferation and differentiation of mesenchymal stem cells. Essentials of Mesenchymal Stem Cell Biology and Its Clinical Translation. Springer, Dordecht, The Netherlands, 75-100.

Li RD, Deng ZL, Hu N, Liang X, et al. (2012). Biphasic effecs of TGF $\beta 1$ on BMP9-induced osteogenic differentiation of mesenchymal stem cells. BMB Rep. 45: 509-514.

Liu Y, Berendsen AD, Jia S, Lotinum S, et al. (2012). Intracellular VEGF regulates the balance between osteoblast and adipocyte differentiation. J. Clin. Invest. 122: 3101-3113.

Luchetti F, Canonico B, Bartolini D, Arcangeletti M, et al. (2014). Melatonin regulates mesenchymal stem cell differentiation: a review. J. Pineal Res. 56: 382-397.

Luther G, Wagner ER, Zhu G, Kang Q, et al. (2011). BMP-9 induced osteogenic differentiation of mesenchymal stem cells: molecular mechanism and therapeutic potential. Curr. Gene Ther. 11: 229-240.

Madl CM, Mehta M, Duda GN, Heilshorn SC, et al. (2014). Presentation of BMP-2 mimicking peptides in 3D hydrogels directs cell fate commitment in osteoblasts and mesenchymal stem cells. Biomacromolecules 15: 445-455.

Patel DM, Shah J and Srivastava AS (2013). Therapeutic potential of mesenchymal stem cells in regenerative medicine. Stem Cells Int. 2013: 496218. 
Wang Y, Hong S, Li M, Zhang J, et al. (2013). Noggin resistance contributes to the potent osteogenic capability of BMP9 in mesenchymal stem cells. J. Orthop. Res. 31: 1796-1803.

Williams SA, Maecker HL, French DM, Liu J, et al. (2011). USP1 deubiquitinates ID proteins to preserve a mesenchymal stem cell program in osteosarcoma. Cell 146: 918-930.

Wu N, Zhao Y, Yin Y, Zhang Y, et al. (2010). Identification and analysis of type II TGF-beta receptors in BMP-9-induced osteogenic differentiation of C3H10T1/2 mesenchymal stem cells. Acta Biochim. Biophys. Sin. (Shanghai) 42: 699708.

Xu DJ, Zhao YZ, Wang J, He JW, et al. (2012). Smads, p38 and ERK1/2 are involved in BMP9-induced osteogenic differentiation of C3H10T1/2 mesenchymal stem cells. BMB Rep. 45: 247-252.

Zhao YF, Xu J, Wang WJ, He JW, et al. (2013). Activation of JNKs is essential for BMP9-induced osteogenic differentiation of mesenchymal stem cells. BMB Rep. 46: 422-427. 